\title{
OUTER MEMBRANE PERMEABILITY OF LIPOPOLYSACCHARIDE DEFICIENT MUTANTS OF SALMONELLA TYPHIMURIUM
}

\author{
D. M. SIRISENA \\ Department of Botany, University of Kelaniya, Kelaniya.
}

(Received: 28 May 1998 ; accepted: 10 August 2000)

\begin{abstract}
In gram negative hacteria, lipopolysaccharide is a major component of the outer membrane permeability barrier against hydrophobic compounds and other molecules. Mutants which produce normal lipopolysaccharide and defective lipopolysaccharide under different growth conditions are useful for comparative studies to understand the nature of this permeability barrier. In this study the outer membrane permeability of temperature-sensitive mutants for lipopolysaccharide biosynthesis was compared at permissive and restrictive temperatures. Two mutants, one producing lipopolysaccharide of chemotype Rc at $30^{\prime \prime} \mathrm{C}$, and another producing more defective deep-rough lipopolysaccharide at $42^{\circ} \mathrm{C}$, and their galE ${ }^{+}$derivatives producing complete (smooth) and deep-rough lipopolysaccharide at $30^{\circ} \mathrm{C}$ and $42^{\prime \prime} \mathrm{C}$ respectively. were used for this study. Their permeability to certain hydrophobic antihiotics, sodium deoxycliolate and sodium dodecyl sulphate was examined by sensitivity disc tests and cell lysis tests. All mutants showed an increased sensitivity to these agents at high temperature. Permeahility to heta-lactam antibiotics was further tested hy heta-lactamase assay carried out with the same mutants harboring a plasmid which makes them to produce the enzyme beta-lactamase. Results of this assay and the gentian violet uptake experiment also indicated the increased permeability to beta-lactam antibiotics and the dye at high temperature, when the lipopolysaccharide becomes more defective. However, in the presence of $\mathrm{Mg}^{2+}$ ions there was no significant difference in their permeability at low and high temperatures. Thus, despite defective lipopolysaccharide structure, $\mathrm{Mg}^{2+} \mathrm{ions}$ seem to restore the barrier function of these mutants to a certain extent.
\end{abstract}

Key words: Lipopolysaccharide, mutants, outer memhrane, permeability, Salmonell.u, temperature-sensitive mutants.

\section{INTRODUCTION}

The outer membrane of gxam negative bacteria functions as an effective permeability barrier against antibiotics, detergents and other molecules. Lipopolysaccharide (LPS), a major component of the outer membrane is mainly responsible for this barrier function. ${ }^{1,2}$ LPS covers approximately $75 \%$ of the outer surface of the outer membrane, while the rest is mainly covered by porin proteins that form small channels for hydrophobic diffusion through the outer membrane. ${ }^{3}$

LPS molecule is composed of four domains; lipid A, inner core oligosaccharide with two unusual sugars i.e. heptose and 2-keto-3-deoxy-octulosonic acid (KDO), the outer core oligosaccharide, and O-antigenic polysaccharide (Fig.1). The negative charge of the LPS molecule is due to the phosphate residues in lipid A and 
the carboxyl groups in $\mathrm{KDO}$. Divalent cations $\left(\mathrm{Ca}^{2+}\right.$ and $\mathrm{Mg}^{2+}$ ) probably link the LPS molecules through ionic bridges to form a highly ordered structure. Presumably, this structure of the LPS layer of the outer membrane explains why the outer membraneacts as an effective permeability barrier. ${ }^{3,4}$ The role of LPS in the barrier function has been studied using mutants which are defective in the synthesis of different domains of the LPS molecule. The lipid A and inner core of the LPS are the regions which are crucial for the barrier function. The lipid A mutants so far characterized are defective in barrier function and susceptible to hydrophobic antibiotics. ${ }^{5}$

Mutants with defective inner core produce truncated LPS and they are highly sensitive to hydrophobic compounds. ${ }^{5}$. These mutants produce uncharged or even increased amounts of LPS. ${ }^{5}$ However, the exact role of LPS inner core in the outer membrane permeability barrier function has yet to be determined.

Comparison of wild type and mutant strains has been the approach taken in many studies to understand the nature of the outer membrane permeability barrier. We have isolated and characterized a new class of Salmonella typhimurium. LT2 mutants which are temperature sensitive for the synthesis of LPS. ${ }^{7}$ These mutants produce LPS containing only the inner core and the lipid $A$ at permissive temperature $\left(30^{\circ} \mathrm{C}\right)$, and LPS lacking the inner core at restrictive temperature $\left(42^{\circ} \mathrm{C}\right)$. Comparison of outer membrane permeability of such mutants at permissive and restrictive temperatures provides more direct information about the role of the inner core domain of LPS as a component of the permeability barrier. This paper deals with the permeability of these mutants to hydrophobic compounds and detergents at permissive and restrictive temperatures.

\section{METHODS \& MATERIALS}

Bacterial strains: Bacterial strains used in this study are listed in Table 1. L-broth and L-agar were used as the routine growth media and they were supplemented with required components for different permeability tests.

Temperature-sensitive $(t s)$ strains used in this study were isolated from a S. typhimurium strain which has a mutation in the galE gene. ${ }^{7}$ This strain is therefore unable to synthesize the enzyme UDP-glucose-4-epimerase which is essential for galactose fermentation. ${ }^{8}$ Due to this defect it produces LPS of chemotype Rc (Fig 1). Such LPS molecules lack the outer core and O-antigen, and the cells have the rough phenotype. The two mutant strains, SA2903 and SA2904, derived from this galE strain also produce LPS of chemotype Rc at $30^{\circ} \mathrm{C}$, but more defective LPS at $42^{\circ} \mathrm{C} .{ }^{7}$ Thus, at high temperature mutants show the "deep rough" phenotype (Fig. 1). To eliminate the influence of the galE mutation on the biosynthesis of LPS, the galE+ allele was transduced into these mutant 
strains. The galE+ transductants from SA2903 and SA2904 were designated SA3046 and SA3047 respectively. These two galE ${ }^{+}$derivatives are also temperature sensitive for LPS synthesis; complete (smooth) LPS at $30^{\circ} \mathrm{C}$ and deep rough LPS at $42^{\circ} \mathrm{C}$. S. typhimurium strains SL3770 and SA1377 which produce smooth and deep rough LPS respectively were used as controls.

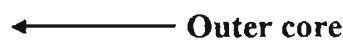

Inner core

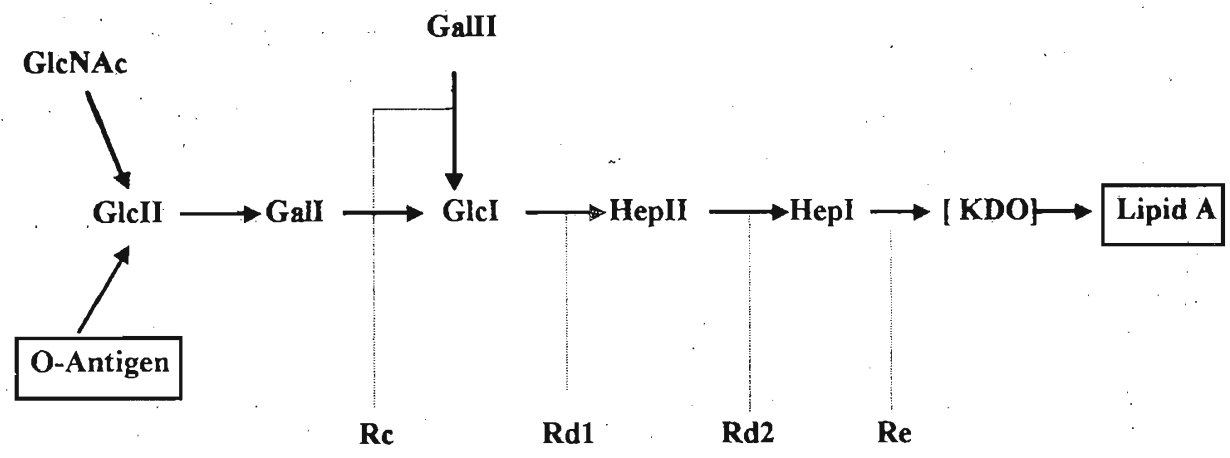

Figure 1: Structure of the lipopolysaccharide of S. typhimurium. $\mathrm{KDO}=2 \mathrm{keto}-$ 3-deoxyoctulosonic acid; GlcNAc= N-acetylglucosamine; Gal= galactose; Glu= glucose; Hep = heptose. Rc, Rd1, Rd2 and Re are chemotypes of LPS produced by mutants of the indicated class (i.e. for LPS completed up to the indicated block)

These bacterial strains were kindly donated by Prof. K. E. Sanderson at the Salmonella Genetic Stock Center, University of Calgary, Alberta, Canada.

Sensitivity disc tests: Sensitivity to différent antibiotics and detergents was tested by placing discs containing known quantities of each compound on bacterial lawns prepared on L-agar. After incubation for $24 \mathrm{~h}$, the size of the zone of inhibition around each disc was measured. The effect of the presence of $\mathrm{Mg}^{2+}$ ions was also tested by using the bacterial cultures grown in L-broth containing $20 \mathrm{mM} \mathrm{MgCl}_{2}$ for preparation of lawns on L-agar containing $20 \mathrm{mM} \mathrm{MgCl}_{2}$.

Sodium deoxycholate (DOC) and sodium dodecyl sulphate (SDS) discs were prepared by dispensing the given amount of each compound onto the filter paper discs. Antibiotic discs were from Becton Dickinson Canada. 
Gentian violet uptake: A method which has been described previously" was modified to determine the uptake of the dye gentian violet. Bacterial cultures were grown to the log phase (50 Klett units, Klett Summersion colorimeter) in L-broth at $30^{\circ} \mathrm{C}$ or $42^{\circ} \mathrm{C}$. The optical density of all cell suspensions was then adjusted to 0.45 at $640 \mathrm{~nm}$ using an LKB Ultrospec-4050 spectrophotometer. The cell suspension $(0.5 \mathrm{ml})$ was mixed with $0.5 \mathrm{ml}$ of aqueous gentian violet $(20 \mu \mathrm{g} / \mathrm{ml})$ and incubated at $37^{\circ} \mathrm{C}$ for $15 \mathrm{~min}$. The tube was chilled in ice and centrifuged in a microcentrifuge. Absorbance of the supernatant at $590 \mathrm{~nm}$ was measured and the dye uptake was calculated based on the estimated amount of dye left in the supernatant.

Table 1: S. typhimurium strains used in this study

\begin{tabular}{ll}
\hline Strain & \multicolumn{1}{c}{ Description \& Reference } \\
SL3770 & Wild type. Produces complete (smooth) LPS \\
LB5010 & gal $\mathrm{E}^{5}$ mutant producing LPS of chemotype Rc. ${ }^{15}$ \\
SA2903 & A temperature sensitive mutant of LB5010. Produces \\
& rough LPS at $30^{\circ} \mathrm{C}$ and deep rough LPS at $42^{\circ} \mathrm{C}^{7}$ \\
SA2904 & same as SA2903 \\
SA3046 & gal $\mathrm{E}^{+}$derivative of SA2903. Produces smooth LPS at \\
& $30^{\circ} \mathrm{C}$ and deep rough LPS at $42^{\circ} \mathrm{C}^{7}$ \\
SA3047 & gal $\mathrm{E}^{+}$derivative of SA2904. Produces smooth LPS at \\
& $30^{\circ} \mathrm{C}$ and deep rough LPS at $42^{\circ} \mathrm{C}^{7}$ \\
SA1377 & A mutant producing deep rough LPS of chemotype Re. ${ }^{16}$ \\
\hline
\end{tabular}

Rate of cell lysis by detergents: Cells grown in L-broth to mid-log phase were washed with $0.07 \mathrm{M}$ phosphate buffer $(\mathrm{pH} 7.2)$ and re-suspended in the same buffer. The $\mathrm{OD}_{640}$ of the cell suspension was adjusted to 0.5 and $1.9 \mathrm{ml}$ of this suspension was incubated in a cuvette at $37^{\circ} \mathrm{C}$ for $30 \mathrm{~min}$. Then $0.1 \mathrm{ml}$ of $10 \%$ sodium deoxycholate (DOC) solution was added to yield a final concentration of $0.5 \%$, and the decrease in absorbance was continually monitored in a LKB Ultrospec- 4050 spectrophotometer. The rate of cell lysis by sodium dodecyl sulphate was also determined in the same manner.

Determination of the outer membrane permeability by B-lactamase assay: Permeability of the outer membrane to several $\beta$-lactam antibiotics was determined as described by Zimmerman and Rosselet. ${ }^{10} \beta$-lactamase assay is based on the ability of bacterial cells to hydrolyse $\beta$-lactam antibiotics once they are transported 
into the periplasmic space. In order to make the bacterial strains synthesize $\beta$-lactamase the plasmid $R 68.45$ which carries the genes for resistance to several $\beta$-lactam antibiotics was introduced into each strain by conjugation. Pseudomonas acruginosa PAO25 carrying R68.45 ${ }^{11}$ was used as the donor in the three way cross.

The enzyme activity of intact cells and sonicated cells was determined by the microiodometric method. ${ }^{12}$ The reaction mixture contained $1 \mathrm{ml}$ of starch-iodine solution, $0.9 \mathrm{ml}$ of phosphate buffer $(0.1 \mathrm{M}, \mathrm{pH} 7.2), 0.1 \mathrm{ml}$ of cell suspension (intact or sonicated) and $1 \mathrm{ml}$ of $0.2 \mathrm{mM} \beta$-lactam antibiotic. Following addition of the antibiotic absorbance at $620 \mathrm{~nm}$ was monitored in a recording spectrophotometer. Absorbance decreases as the starch-iodine complex is reduced when $\beta$-lactamase hydrolyzes the antibiotic. Thus, the rate of hydrolysis of $\beta$-lactam antibiotics was determined by following the rate of decolorization of starch-iodine complex. The ratio between the rate of hydrolysis of $\beta$-lactam antibiotics by intact cells and sonicated cells $\left(\mathrm{Vi} / \mathrm{Vs}_{\mathrm{s}}\right)$ was used as a measure of permeability.

Bacterial cells were grown in L-broth at $30^{\prime \prime} \mathrm{C}$ or $42^{\circ} \mathrm{C}$ and the enzyme assay was done at room temperature.

\section{RESULTS}

\section{Sensitivity to antibiotic and detergent discs:}

The two ts mutants, SA2903 and SA2904, and their galE ${ }^{+}$derivatives, SA3046 and SA3047, were tested for their sensitivity to different antibiotics and detergents at permissive and restrictive temperatures. When grown in L-agar all four strains showed an increased sensitivity to highly hydrophobic antibiotics; i.e. novobiocin, oleandomycin and nafcillin (Table 2). The wild type strain SL3770, which produces complete LPS did not show any sensitivity to these antibiotics. The $t s$ mutants produced much larger zones of inhibition at $42^{\circ} \mathrm{C}$ than at $30^{\prime \prime} \mathrm{C}$ indicating their increased permeability to these drugs when the LPS structure becomes more defective at the high temperature. Similar results were obtained with sodium deoxycholate (DOC) and sodium dodecyl sulphate (SDS).

There was a dramatic decrease in the level of sensitivity when the test was done in the medium containing $\mathrm{Mg}^{2+}$ ions. All four strains became completely resistant to oleandomycin $(15 \mu \mathrm{g})$ at both temperatures in the presence of $\mathrm{Mg}^{2+}$ ions (Table 2).

\section{Gentian violet uptake by bacterial cells:}

When grown in L-broth, gentian violet uptake by SA2903 and SA2904 was doubled at the restrictive temperature (Table 3). Addition of $\mathrm{MgCl}_{2}$ into the growth medium 
drastically reduced the dye uptake. In fact, compared to the dye uptake of the wild type strain there was no indication of active dye uptake by the mutants at low temperature (Table 3). Similar results were obtained with the strains SA3046 and SA3047.

Table 3: Uptake of gentian violet by wild type and different LPS-deficient $t s$ mutant strains of S. typhimurium.

\begin{tabular}{|c|c|c|c|c|c|c|}
\hline \multirow{3}{*}{ Strain } & \multirow{2}{*}{\multicolumn{2}{|c|}{$\begin{array}{c}\text { LPS } \\
\text { Phenotype }\end{array}$}} & \multicolumn{4}{|c|}{$\%$ uptake } \\
\hline & & & \multicolumn{2}{|c|}{ L-broth } & \multicolumn{2}{|c|}{$\mathrm{L}+\mathrm{MgCl}_{2}$} \\
\hline & $30^{\prime \prime}$ & $42^{\prime \prime}$ & $30^{\circ}$ & $42^{\prime \prime}$ & $30^{n}$ & $42^{\circ}$ \\
\hline SL3770 & S & $\mathrm{S}$ & 5.2 & 5.2 & 4.8 & 1.1 \\
\hline SL2903 & $\mathrm{R}$ & DR & 30.7 & 57.6 & 4.9 & 20.0 \\
\hline SA2904 & $R$ & $\mathrm{DR}$ & 26.0 & 53.8 & 4.0 & 9.8 \\
\hline SA3046 & S & $\mathrm{DR}$ & 23.7 & 39.1 & 1.9 & 10.6 \\
\hline SA3047 & $S$ & $\mathrm{DR}$ & 20.1 & 42.7 & 3.4 & 7.9 \\
\hline SA1377 & $\mathrm{DR}$ & $\mathrm{DR}$ & 49.8 & 39.7 & 22.6 & 9.0 \\
\hline
\end{tabular}

a - Percentage of gentian violet taken up by cells when the dye was added to a tinal concentration of $10 \mu \mathrm{g} / \mathrm{ml}$. Each value is an average of three independent tests.

b - S= Smooth (Complete) LPS, R= Rough LPS; DR= Deep rough LPS

\section{Cell lysis by detergents (DOC and SDS):}

In the lysis test performed with DOC, the $t$ s strain SA3047 grown in L-broth at $42^{\circ} \mathrm{C}$ showed a dramatic drop in optical density indicating a very rapid lysis (Fig. 2), which was similar to lysis pattern of the deep rough strain SA1377. Cells of SA3047 grown at $30^{\circ} \mathrm{C}$ lysed slowly. Thus, as seen in Figure 2, there is a very clear difference between the rates of lysis of SA3047 cells grown at low and high temperatures. For the same strain the rates of cell lysis obtained with SDS were higher than those obtained with DOC (Fig. 3). However, a similar pattern of cell lysis was observed with SDS too. Thus, the increased sensitivity of this $t s$ mutant strain to the two anionic detergents at the restrictive temperature was further confirmed.

The rate of lysis of SA3047 cells grown in the presence of $\mathrm{Mg}^{2+}$ ions showed a clear decrease compared to that of the cells grown without $\mathrm{Mg}^{2+}$ ions (Fig.2). As such, the difference between the rates of lysis of these $t s$ mutant cells grown at $30^{\circ} \mathrm{C}$ and $42^{\circ} \mathrm{C}$ was not dramatic when they were grown in the medium containing $\mathrm{Mg}^{2+}$ ions. 


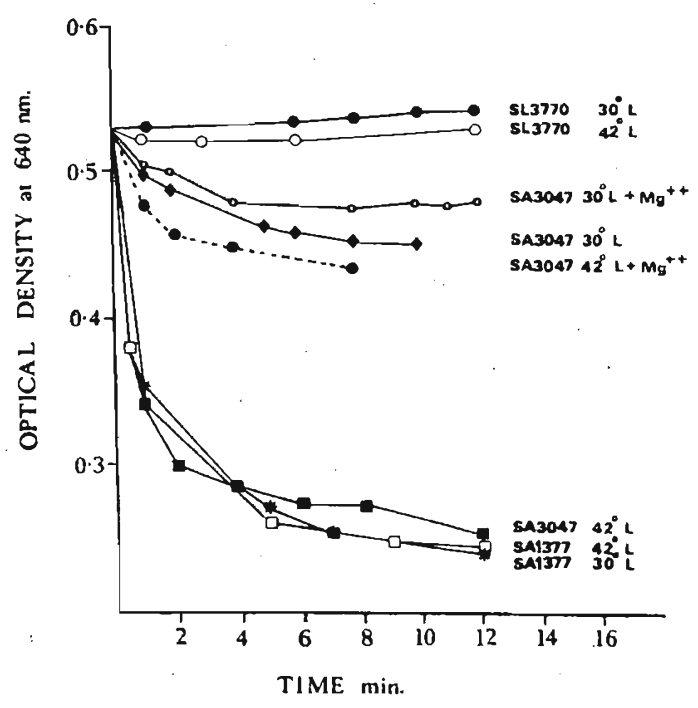

Figure 2: Rates of cell lysis by sodium deoxycholate. Cells grown at $30^{\circ} \mathrm{C}$ and $42^{\circ} \mathrm{C}$ in L-broth with or without $\mathrm{MgCl}_{2}$ were lysed by $\mathrm{DOC}(0.5 \%)$. Strain, growth temperature and medium are given at the end of each curve.

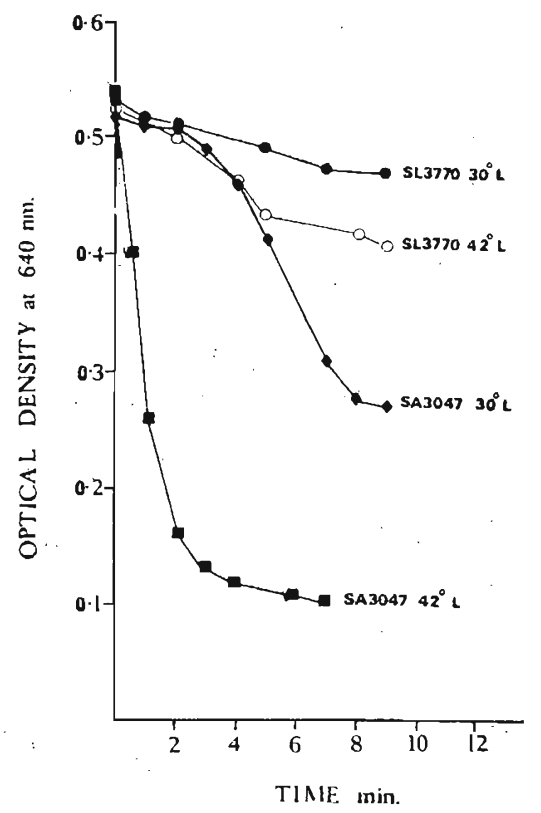

Figure 3: Rates of cell lysis by sodium dodecyl sulphate. Cells grown at $30^{\circ} \mathrm{C}$ and $42{ }^{\circ} \mathrm{C}$ in L-broth were lysed by $\operatorname{SDS}(0.5 \%)$. Strain and growth temperature are given at the end of each curve. 


\section{B-lactamase assay:}

Results of the $\beta$-lactamase assay are also in agreement with those of other permeability tests. The $t s$ strains which were made to synthesize periplasmic B-lactamase by introducing the plasmid R68.45 were able to hydrolyze piperacillin and carbenicillin at high rates at $42^{\circ} \mathrm{C}$ compared to the rates obtained with the cells grown at $30^{\circ} \mathrm{C}$ (Table 4). The strain with complete LPS (SL3770/R $\mathrm{R}^{+}$) and the deep rough strain SA1377/ $\mathrm{R}^{+}$did not show an increase in the rate of hydrolysis of these antibiotics at high temperature (Table 4).

Table 4: Rates of hydrolysis of $\beta$-lactam antibiotics by intact cells ( $V$ intact) and sonicated cells ( $V$ sonicated) expressed as ( $V$ intact/ $V$ sonicated $)^{\text {n. }}$.

\begin{tabular}{|c|c|c|c|c|c|c|}
\hline \multirow{3}{*}{ Strain } & \multicolumn{6}{|c|}{$\beta$-lactam antibiotic } \\
\hline & \multicolumn{2}{|c|}{ Piperacillin } & \multicolumn{2}{|c|}{ Ticarcillin } & \multicolumn{2}{|c|}{ Carbenicillin } \\
\hline & $30^{\prime \prime}$ & $42^{\circ}$ & $30^{\prime \prime}$ & $42^{\prime \prime}$ & $30^{\prime \prime}$ & $42^{\prime \prime}$ \\
\hline SL3770/R+ & 0.012 & 0.004 & 0.055 & 0.032 & 0.065 & 0.159 \\
\hline $\mathrm{SA} 2903 / \mathrm{R}^{+}$ & 0.007 & 0.07 & 0.029 & 0.057 & 0.038 & 0.078 \\
\hline $\mathrm{SA} 2904 / \mathrm{R}^{+}$ & 0.02 & 0.073 & 0.031 & 0.019 & 0.083 & 0.096 \\
\hline $\mathrm{SA} 1377 / \mathrm{R}^{+}$ & 0.028 & 0.031 & 0.074 & 0.026 & 0.093 & 0.019 \\
\hline
\end{tabular}

a - Rate is expressed as $\mu \mathrm{mol} \mathrm{min}^{-1} \mathrm{mg}^{-1}$ of dry weight of cells

\section{DISCUSSION}

In S. typhimurium and several other gram negative bacteria LPS plays a major role in the barrier function of their outer membrane. Wild type strains which are completely resistant to certain antibiotics and other hydrophobic compounds, become sensitive to such agents when the LPS structure is defective. S. typhimurium mutants which are defective in the synthesis of the inner core domain contain only the lipid A (Fig.1). These mutants are highly sensitive to hydrophobic agents because their outer membrane permeability barrier is breached when the LPS structure becomes defective. ${ }^{1,2}$ However, the exact role of LPS in the barrier function has to be determined yet. Comparison of different classes of LPS mutants with wild type or less defective LPS mutant strains for their outer membrane permeability has been the approach taken in previous studies. Use of temperature sensitive mutants that produce two different LPS phenotypes at permissive and restrictive temperatures allows a more appropriate comparison of their barrier function rather than using different strains with different LPS phenotypes. This is 
the first report on comparison of outer membrane permeability using such $t s$ mutants of S. typhimurium with defects in the inner core region of LPS.

Results of the sensitivity disc test indicated the breach of the permeability barrier of these $t s$ mutants at high temperature (Table 2), when they were grown in L-broth. The galE ${ }^{+}$derivatives of the $t s$ mutants, SA3046 and SA3047, produce smooth (wild type) LPS at low temperature. ${ }^{7}$ However, they are not completely resistant to the tested antibiotics except bacitracin.

Although gentian violet uptake has been used as a reliable indicator of the outer membrane permeability, about $20 \%$ of the dye added to the medium binds to the surface of the cells regardless of the nature of their outer membrane." Thus, uptake values of $20 \%$ or less represent this passive uptake. The comparison of uptake values in $t s$ mutants indicated that their dye uptake is greatly dependent on the growth temperature, and therefore, on the LPS chemotype. As shown by the results of the gentian violet uptake experiment given in Table 3 , at high temperature the two $t s$ mutants and their galE ${ }^{+}$derivatives grown in L-broth have taken up as much as double the amount of dye they absorb at low temperature. Despite the passive binding to a certain extent, these results clearly demonstrate the increased permeability of the cells when the LPS structure becomes more defective. Similar results have been obtained for dye uptake by deep rough S.typhimurium mutants. ${ }^{6}$

Assay of $\beta$-lactamase activity is a more direct measure of permeability to $\beta$-lactam antibiotics. Although the results of the $\beta$-lactamase assay was not very pronounced the two ts mutants show the correlation between defective LPS structure and the outer membrane permeability (Table 4). Cells of the strains SA2903/R+ and SA2904/R+ have an increased permeability to piperacillin and carbenicillin at high temperature. However, a clear difference in $(\mathrm{Vi} / \mathrm{Vs})$ ratio at two temperatures was not observed with ticarcillin. This may be due to either the fact that ticarcillin is not a good substrate for this enzyme or its high lipophilic character compared to other $\beta$-lactam antibiotics. ${ }^{10}$ Comparison of rates of hydrolysis with the strains SL3770/R+ and SA1377/R+ (Table 4) suggests that the difference in the rates of hydrolysis may not be solely attributed to their LPS structure.

The most convincing and unambiguous data to demonstrate the varying level of outer membrane permeability of ts mutants came from the spectrophotometrically determined rates of cell lysis by detergents. The level of sensitivity to DOC is directly related to the LPS chemotype, because the wild type strain is completely resistant to DOC while the $t s$ mutant SA3047 grown at $42^{\circ} \mathrm{C}$, and the deep rough strain SA1377 are extremely sensitive (Fig.2). 
It has been shown that cations such as $\mathrm{Mg}^{2+}, \mathrm{Ca}^{2+}$ and $\mathrm{Na}^{+}$have a profound effect on the permeability properties of the gram negative outer membrane. Divalent cations such as $\mathrm{Ca}^{2+}$ and $\mathrm{Mg}^{2+}$ are important for organizing the outer membrane. Removal of such cations facilitates the passage of hydrophobic molecules across the outer membrane, and the addition of $\mathrm{Mg}^{2+}$ seems to reverse such permeability changes. ${ }^{13}$ In LPS mutants of $S$. typhimurium the uptake of hydrophobic compounds is reduced in the presence of $\mathrm{Mg}^{2+}$ ions. ${ }^{6}$ Therefore, all experiments on the permeability of the two ts mutants and their galE+ derivatives were also performed in the presence of $\mathrm{Mg}^{2+}$ ions.

All four strains showed a substantial decrease in their sensitivity to hydrophobic compounds in the presence of $\mathrm{Mg}^{2+}$ ions (Table 2,3). The restoration of the barrier layer to a greater extent by $\mathrm{Mg}^{2+}$ ions was quite clear in the dramatic drop in gentian violet uptake and the decreased rate of cell lysis by DOC (Fig. 2). This suggests that in these mutants the breach of the permeability barrier at high temperature is not solely due to their defective LPS structure.

Different experimental approaches used in this study confirmed the increased permeability of the LPS inner core mutants to hydrophobic compounds. However, the cell lysis method used for the first time in this study appears to be a better way of comparing the permeability barrier function. Since the results obtained with these temperature sensitive LPS deficient mutants are in agreement with previous reports, they are valuable for further comparative studies to understand the nature of the permeability barrier of gram negative bacteria.

\section{References}

1. Nilkaido H. \& Vaara M. (1985). Molecular basis of bacterial outer membrane permeability. Microbiological Reviews 49:1-32.

2. Hancock R. E. W. (1984). Alterations in outer membrane permeability. Annual Review of Microbiology 38: 237-264.

3. Nikaido H. (1996). Outer membrane. In: Escherichia coli and Salmonella. Vol. 2 Cellular and molecular biology (Ed. F.C. Neidhardt) pp.29-47. American Society for Microbiology, Washinton D.C.

4. Vaara M. (1993). Antibiotic-supersusceptible mutants of Escherichia coli and Salmonella typhimurium. Antimicrobial Agents and Chemotherapy 37:2255-2260.

5. Roantree R. J., Kuo T. T. \& MacPhee D. G. (1977). The effect of defined lipopolysaccharide core defects upon antibiotic resistance of Salmonella typhimurium. Journal of General Microbiology 103: 223-234. 
6. Stan-Lotter H., Gupta M. \& Sanderson K. E. (1979). The influence of cations on the permeability of the outer membrane of Salmonella typhimurium and other gram negative bacteria. Canadian Journal of Microbiology 25: 475-485.

7. Sirisena D. M. \& Sanderson K. E. (1994). Temperature-sensitive, lipopolysaccharide-deficient mutants of Salmonella typhimurium. World Journal of Microbiology \& Biotechnology 10: 681-685.

8. Nikaido H. (1962). Studies on the biosynthesis of cell wall polysaccharide in mutant strains of Salmonella. Proceedings of the National Academy of Sciences (USA) 48: 1542-1548.

9. Gustafsson P., Nordstrom K. \& Normark S. (1973). Outer penetration barrier of Escherichia coli K-12: kinetics of the uptake of gentian violet by wild type and envelope mutants. Journal of Bacteriology 116: 893-900.

10. Zimmermann W. \& Rosselet A. (1977). Function of the outer membrane of Escherichia coli as a permeability barrier to beta-lactam antibiotics. Antimicrobial Agents and Chemotherapy 12: 368-372.

11. Godfrey A. J. \& Bryan L. E. (1984). Resistance of Pseudomonas aeruginosa to new beta-lactamase - resistant beta-lactams. Antimicrobial Agents and Chemotherapy 26: 485-488.

12. Ross G. W. \& O'callaghan C. H. (1975). Beta-lactamase assays. Methods in Enzymology 43: 31-88.

13. Chatterjee A. K., Ross H. \& Sanderson K. E. (1976). Leakage of periplasmic enzymes from lipopolysaccharide - deficient mutants of Salmonella typhimurium. Canadian Journal of Microbiology 22: 1549-1560.

14. Vaara M. (1992). Agents that increase the permeability of the outer membrane. Microbiolological Reviews 56: 395-411

15. Bullas L. R. \& Ryu J. (1983). Salmonella typhimurium LT2 strains which are $\mathrm{r} \cdot \mathrm{m}$ for all three chromosomally located systems of DNA restriction and modification. Journal of Bacteriology 156:471-474.

16. Sanderson K. E., Wyngaarden J. V., Luderitz O. \& Stocker B. A. D. (1974). Rough mutants of Salmonella typhimurium with defects in the heptose region of the lipopolysaccharide core. Canadian Journal of Microbiology 20:1127-1134. 